\title{
Knot detection in X-ray CT images of wood
}

\author{
A. Krähenbühl ${ }^{1}$, B. Kerautret ${ }^{1}$, I. Debled-Rennesson ${ }^{1}$, F. Longuetaud ${ }^{2}$ and F. \\ Mothe $^{2}$ \\ 1 Université de Lorraine, LORIA, Adagio team, UMR 7503, 54506, Nancy, France \\ 2 INRA, UMR1092 LERFoB, 54280 Champenoux, France
}

\begin{abstract}
This paper presents an original problem of knot detection in 3D X-ray Computer Tomography images of wood stems. This image type is very different from classical medical images and presents specific geometric structures. These ones are characteristic of wood stems nature. The contribution of this work is to exploit the original geometric structures in a simple and fast algorithm to automatically detect and analyze the wood knots. The proposed approach is robust to different wood qualities, like moisture or noise, and more simple to implement than classical deformable models approaches.
\end{abstract}

\section{Introduction}

Detect and identify automatically digital objects in $3 \mathrm{D}$ volumetric images is a challenge, especially in medical imaging applications. Various methods are proposed to extract and measure internal characteristics of the human, like brain or blood vessel. Currently, the most effective techniques use deformable models [1].

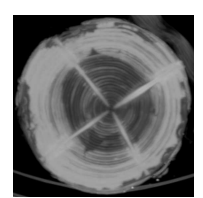

(a)

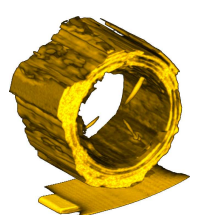

(b)

Fig. 1. Example of wood image and $3 \mathrm{D}$ volumic rendering.

In this work, we focus on a similar problem with 3D digital images of wood. The images are obtained by medical X-Ray Computer Tomography (CT) scanners and are illustrated on Fig. 1. On Fig. 1(a), a raw image is projected in an intensity interval which highlight four knots starting from the $\mathrm{pith}^{3}$. The related volume obtained by thresholding is rendered on Fig. 1(b).

A knot as in Fig. 1 and Fig. 2 is the first part of a branch, included within the tree stem due to the radial growth of the stem. The frequency and size of the knots are the first depreciation factors considered by wood suppliers for estimating the price of timber. Knottiness is also one of the main criteria considered in the visual grading of lumber. Knowledge of knot geometry and location would be valuable at sawmills for optimising cutting decisions or improving the grading of logs. X-ray $\mathrm{CT}$ has been recognised as being the most promising method to non-destructively analyse the internal structure of wood [2]. CT scanners designed expressly for the wood industry are now available and some of

\footnotetext{
${ }^{3}$ pith : central part of stem.
} 
the largest sawmills are now equipped with them. Moreover, such detailed data about knottiness are needed in the field of forest research to study for example the relationship between tree growth conditions and wood quality.

Several methods were proposed for measuring knottiness on the basis of CT images (see [3] for a review). But no method is entirely automatic and well adapted to all species of wood and to wood containing wet areas such as sapwood $^{4}$

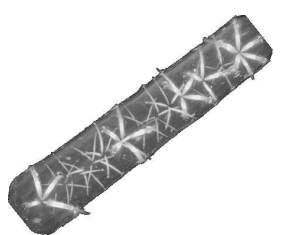

(a) Spruce stem

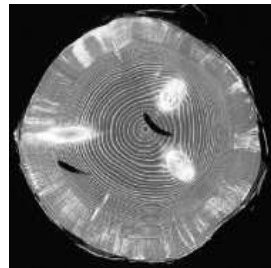

(b) Pinus slice

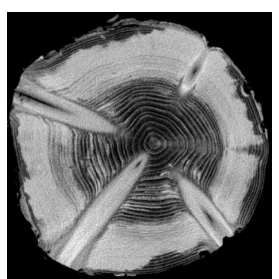

(c) Spruce slice

Fig. 2. X-Ray CT images of stem : (a) 3D volumetric rendering, (b) dry and (c) with sapwood.

We proposed in [3] an entirely automated method to identify knots from X-ray CT images of a piece of wood (log or beam) and obtain data on knot geometry without any human intervention. However, the first step of the algorithm, image segmentation, was not studied in details. A simple thresholding operation was sufficient enough for processing the air-dried wood that were used in this study. For an application in industrial conditions, there is a need to improve the segmentation algorithm, especially for wet wood containing areas of sapwood (see Fig. 2(c)).

The main contribution of this paper is to automatically and quickly detect all knots of a given log based on the analysis of X-ray CT images. Various softwood species are studied, various moisture contents were considered leading to have large sapwood areas visible in the CT images (see Fig. 2(c)), and our method identifies all knots in all cases.

The proposed knot detection is based on a very precise detection of the localisation of each knot. To do this, we use intensity variation between two consecutive slices and study a specific intensity histogram. We proceed in two steps. The first one is to select slice intervals where intensity variations are important around the pith. In the second step, we keep a set of angular sectors also centered on the pith in each interval from the same criterion of intensity variation.

In the next section we list all tools needed by the proposed method. Section 3 introduces the definition of the cumulative $\mathrm{z}$-motion histogram which allows to identify the meaning slice intervals and to deduce the angular sector of each knot. The last section shows results and presents perspectives of this work.

\footnotetext{
${ }^{4}$ sapwood : wood area located at the periphery of the stem.
} 


\section{Suitable tools for X-Ray CT images of logs}

\section{$2.1 \quad$ Pith detection}

Pith detection is an essential first step to wood knot detection. In particular, in the proposed method it will allow to focus on a restricted area around the pith. In this area, we are sure that intensity variations observed in images indicate the presence of knots. Indeed, the knots are connected to the pith where they have their origin. They are oriented radially in a log, from the pith to the bark, and generally upward. Some knots are totally included into the log and covered by the radial growth rings ${ }^{5}$ but in general, thay are still visible at the outside of the log in the form of branches.

To detect pith, we use the algorithm proposed by Fleur Longuetaud [4] and improved by Boukadida et al. [5]. The idea is to detect the pith successively in each slice. Its location in slice $n$ allows to steer and speed up pith computing on slice $n+1$.

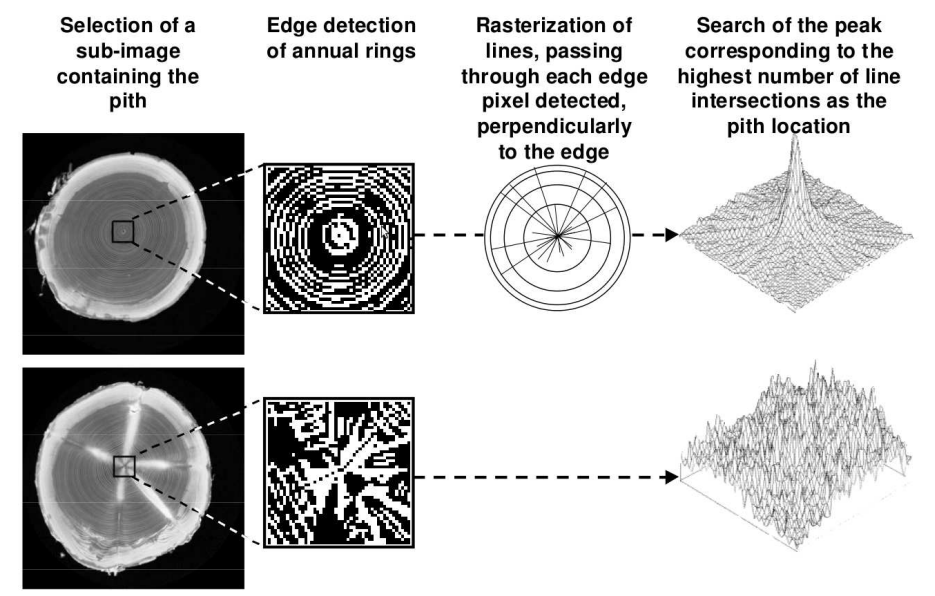

Fig. 3. Pith detection in two slices, with (below) and without (above) knot.

The first step of the algorithm uses the roundness of growth rings. They are detected in each slice $I_{k}$ with a Sobel filter. This one computes $G_{x}$ and $G_{y}$ images, respectively the gradients of $I_{k}$ along $x$ and $y$.

A new image $I_{k}^{\prime}$ is computed with a thresholding of $G$ norm (see Fig. 3). $I_{k}^{\prime}$ allows to define a set of lines. For that, we are computing $\Theta$, the direction of gradient in each pixel of $I_{k}^{\prime}$ not null. After, we are building the map of the intersection locations for all lines taken two by two. The intersection locations are computed with an Hough transform [6]. The pith position is then defined like the location of the largest number of intersecting lines.

In a second step, the pith position is corrected in slices where pith neighbourhood is noisy. It is usually the slices with knots. These ones disturb the roudness

\footnotetext{
${ }^{5}$ growth rings: concentric circles in stem. They are centered on pith.
} 
of growth rings and this noise hugely affects the gradient direction and therefore Hough transform. In these slices, the pith is recomputed by linear interpolation of the previous and the next slice.

\subsection{Intervals detection in an histogram}

The method that we propose detects intervals centered on maximums. It is a problem that can be very difficult to solve according to noise level on histogram. The detection that we are now presenting is suitable for the histograms we have to deal with.

Let $H$ be an $N$-class histogram with $H=\left(H_{k}\right)_{k \in[1, N]}$.

Smoothing The first step consists to smooth $H$ with an averaging filter of radius $r_{f}$. The aim is to improve the computing of the discrete derivative during the following step. We get $H^{l}$ histogram where the $k$ class is :

$$
H_{k}^{l}=\frac{1}{2 r_{f}+1} \sum_{i=k-r_{f}}^{k+r_{f}} H_{i}
$$

with $r_{f}$ : radius of histogram averaging mask.

Maximums computing We defined $M$ as the set of the index of $H^{l}$ maximums from a neighbourhood of $r_{M}$ radius.

$$
M=\left\{k \mid H_{k}^{l}>t_{M} \text { and } \forall i \in\left[-r_{M},-1\right] \cup\left[1, r_{M}\right], H_{k}^{l}>H_{k+i}^{l}\right\}
$$

with $t_{M}$ : minimum value for one maximum.

$r_{M}$ : width of neighbourhood on which the maximums are defined.

Value of a maximum must be greater than $t_{M}$. This allows to avoid the small maximums coming from the noise. And a maximum must have a value greater than the ones of its $\frac{r_{M}}{2}$ left and right neighbours.

Intervals computing Let $V$ be the set of searched intervals. We determine $V$ from the $M$ set of maximum indexes. An interval of classes is computed from each maximum, starting from each side of this maximum.

$$
\begin{aligned}
& V=\{[k-i, k+j] \mid k \in M \\
& \text { and } \exists t_{i} \in[k-i, k] \quad \text { such that }\left\{\begin{array}{l}
\forall t \in\left[t_{i}, k\right], \quad H_{t}^{l}>t_{V} \\
\forall t \in] k-i, t_{i}\left[, H_{t}^{l} \leq t_{V} \text { and }\left(H_{t}^{l}\right)^{\prime}>0\right. \\
\left(H_{k-i}^{l}\right)^{\prime} \leq 0
\end{array}\right. \\
& \text { and } \exists t_{j} \in[k+1, k+j] \text { such that }\left\{\begin{array}{l}
\left.\forall t \in] k, t_{j}\right], \quad H_{t}^{l}>t_{V} \\
\forall t \in] t_{j}, k+j\left[, H_{t}^{l} \leq t_{V} \text { and }\left(H_{t}^{l}\right)^{\prime}<0\right. \\
\left(H_{k+j}^{l}\right)^{\prime} \geq 0
\end{array}\right. \\
& \begin{array}{l}
\text { and } j-i>w_{V} \\
\text { and }
\end{array}
\end{aligned}
$$




$$
\begin{gathered}
\left(H_{t}^{l}\right)^{\prime}=H_{t}^{l}-H_{t-1}^{l} \\
\text { and } t_{V}: \text { derivative threshold. } \\
w_{V}: \text { minimum width of an interval. }
\end{gathered}
$$

Derivatives are tested on each side of maximums only, from the nearest neighbour with a value lower than $t_{V}$. An interval is kept if its magnitude is larger than $w_{V}$.

\section{Z-motion histogram and knot detection}

With detection methods of pith and intervals, presented in previous section, we can determine knot areas. They take the form of angular sectors of cylinder and our goal is to get one area by knot. Let us specify that we detect these areas in image sequences from medical X-Ray scanner. Each image corresponds to a circular slice of log (see Fig. 2). Pixel values corresponds to density on Hounsfield scale. This density is linearly correlated to the wood density. We referred either to wood density or to images intensity.

On X-Ray images of log, pixel intensity is approximately between -3000 and 3000. These bounds changed few from an image to an other. On Hounsfield scale, wood density is usually greater than -900 and always lesser than 530. All image values out of this interval will be considered as outlier and ignored.

Our method consists in applying twice the process of interval detection. The two processed histograms will be constructed from the same notion : the zmotion.

The z-motion We decided to use the z-motion by observing successively an image sequence. By scrolling an image sequence of one log, egg-shaped shapes are observed in motion. These movements occur from pith to bark, orthogonally to the growth rings, when the images are scrolled from the bottom to the top of the tree. These are the knot movements that have their origin at the pith location and have grown radially and upwards.

The z-motion $Z$ of a slice $I_{k}$ is defined like the intensity variation between the two successive slices $I_{k-1}$ and $I_{k}$ :

$$
Z_{k}=\left|I_{k}-I_{k-1}\right|
$$

With help of this notion, we now present the two steps of our method.

Before this two steps, it is necessary to detect pith (see Sec. 2.1) on all slices. It allows to define a $C$ circle centered on pith in each slice of log. Then for the first step, the histogram is constructed by cumulating the z-motion of each slice on the entire circle $C$. We then obtain the $H_{\text {slices }}$ histogram on which we apply the process of interval detection (see Sec. 2.2). During the second step, we subdivided the $C$ circle in a set of angular sectors with same size. For each interval of $H_{\text {slices }}$, we combine the z-motion separately on each angular sector.

Hereafter are more details about the construction of these two histograms. 


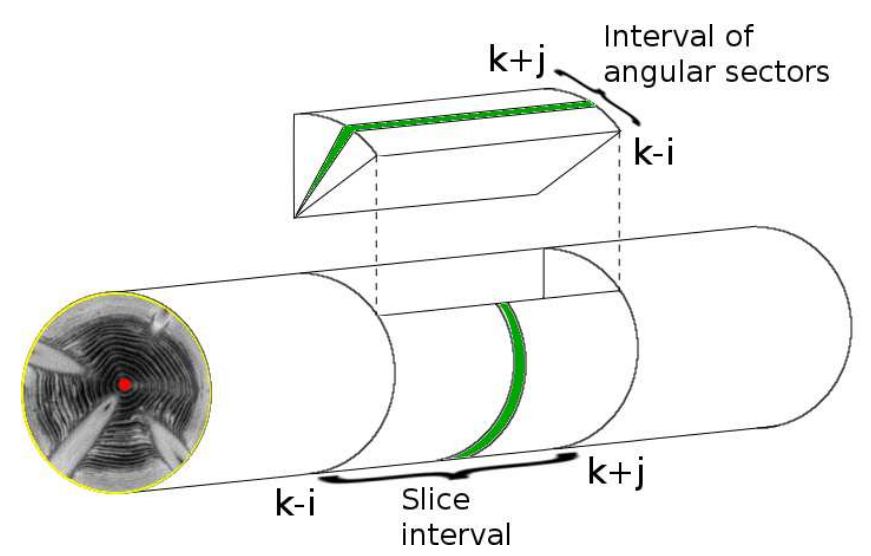

Fig. 4. Angular sesctors intervals on a given slice interval. The green sections represents the slice and the angular sector where the z-motion cumulation is maximum. We can see in initial $\log$ section the considered circle $C$ and the localisation of pith.

\subsection{The histogram of cumulative z-motion}

The slice histogram Let $I$ be a set of images of a $\log$ slice. Let $C$ be a circle of radius $r_{C}$. We defined the $H_{\text {slices }}(k)$ value of $H_{\text {slices }}$ histogram for the $I_{k}$ slice as follows :

$$
H_{\text {slices }}(k)=\sum_{(i, j) \in C} Z_{k}(i, j)
$$

where

- $C$ is centered on the pith position in $I_{k}$ slice.

- $\forall(i, j), I_{k}(i, j) \in\left[i_{\min }, i_{\max }\right]$ et $I_{k-1}(i, j) \in\left[i_{\min }, i_{\max }\right]$

$-\forall(i, j), Z_{k}(i, j) \in\left[z_{\min }, z_{\max }\right]$

with $\quad r_{C}$ : radius of the cumulative z-motion circle.

$\left[i_{\min }, i_{\max }\right]:$ intensity interval.

$\left[z_{\min }, z_{\max }\right]$ : interval of intensity variations.

The z-motion is only computed inside the $C$ circle centered on pith. According to experts, the minimum wood density can be fixed to -900 . It is our value for $i_{\min }$. For $i_{\max }$, we fixed its value to 530, a biological constant for the maximum wood density. Variations lesser than $z_{\min }$ due to growth rings, sapwood, etc. are considered as noise. Variations in $\left[z_{\min }, z_{\max }\right]$ interval are potential movements of knots. Variations greater than $z_{\max }$ are considered as outliers.

In this first step, each histogram value corresponds to the z-motion sum in a slice. Intervals are therefore slice intervals where the cumulative z-motion is important (see Fig. 5).

One of the most important parameters is the $r_{C}$ radius of $C$ circle. It is determine on each slice. We start from pith in ten directions as long as pixel values are greater than -900 . In fact, values of X-ray scanned images fall drastically 


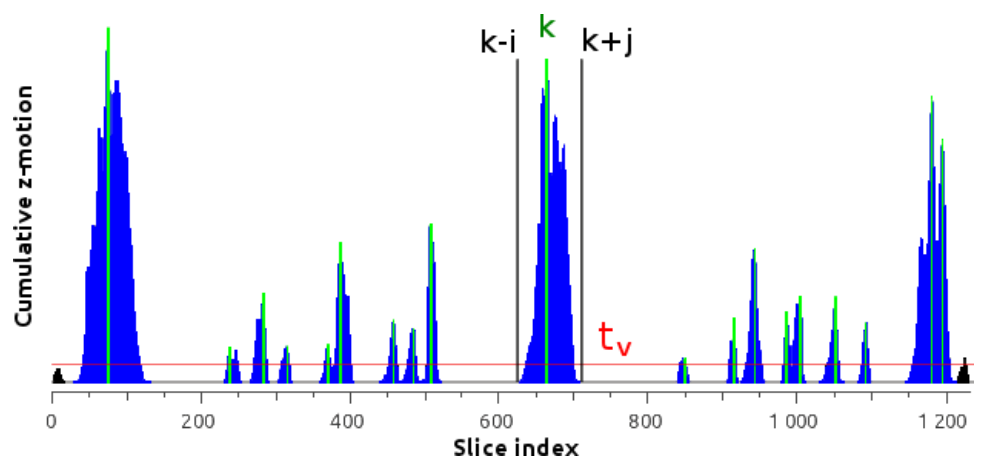

Fig. 5. Histogram after detection of slice intervals in a spruce log.

below -900 when we leave the log. Then we compute the average radius $r$ and $r_{C}$ is fixed to $0.8 \cdot r$.

The z-motion interval $\left[z_{\min }, z_{\max }\right]$ is fixed to $[200,500]$ to just cumulate important z-motion. After histogram construction, it is smoothed with an averaging mask where $r_{f}=2$. Each histogram value becomes candidate to the maximum set if its value is greater than $t_{M}=5 \%$ of the maximum value of $H_{\text {slices }}$.

To compute intervals, we fix the $t_{V}$ threshold to the same value as $t_{M}$. All the first neighbors of a maximum greater than $t_{V}$ belong to the interval of this maximum. From the first neighbor lower than $t_{V}$, following neighbors are added to the initial interval until the discrete derivative goes through 0 . Finally we just keep slice intervals with a width greater than $w_{V}=10$. We obtain a set of slice intervals $V_{H_{\text {slices }}}$ with all knots.

The histogram of angular sectors This second step is applied on each slice interval obtained at previous step. For a given slice interval, we cumulate zmotion separately in each angular sector. We obtain the histogram of cumulative intensity by angular sector (see Fig. 6). Angular sectors are a partition of $C$ circle in $N$ sectors with a same angle. We choose $N=360$.

Let $[a, b] \in V_{\text {slices }}$ be an interval of slice indexes. Let $C$ be a circle with a $r_{C}$ radius split in 360 sectors with same angle. We defined the value $H_{\text {sectors }}(\alpha)$ of $H_{\text {sectors }}$ histogram restricted to angle of $\alpha$ index as follows :

$$
H_{\text {sectors }}(\alpha)=\sum_{k \in[a, b]} \sum_{(i, j) \in C_{\alpha}} Z_{k}(i, j)
$$

with

- $C$ centered on pith coordinates in $I_{k}$

- $C_{\alpha}$ the $C$ angular sector of $\alpha$ index $\in[0,359]$

- $\forall k, \forall(i, j), I_{k}(i, j)>i_{\text {min }}$ et $I_{k-1}(i, j)>i_{\text {min }}$

- $\forall k, \forall(i, j), Z_{k}(i, j) \in\left[z_{\min }, z_{\max }\right]$

For an interval $V_{q}$ of $V_{\text {slices }}$, we obtain an histogram $H_{\text {sector }}$ of z-motion distribution according to angular sector. Like in the first step, we use algorithm of interval detection to obtain a set of angular intervals which contains one knot. 


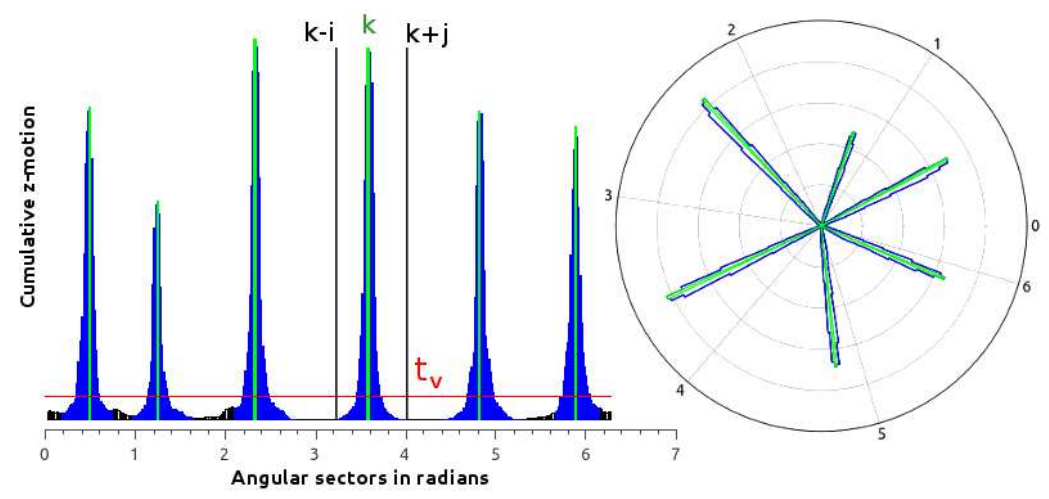

Fig. 6. Histogram and circular projection after detection of sector interval.

We keep the same parameters as at the first step, $t_{M}$ and $t_{V}$ excepted. In fact, the majority of parameters was fixed in studying scanned picture properties and in this second step, images are the same.

However, $t_{M}$ and $t_{V}$ correspond to histogram properties. On histograms of angular sectors, peaks are usually the same size, unlike the slice histograms. In fact, the knots of a slice interval have about about same volume. We fix rationally $t_{M}$ and $t_{V}$ at $50 \%$ of the $H_{\text {sectors }}$ maximum value without risking to forget a maximum and therefore a knot sector.

\section{Results}

The presented method is able to quickly isolate each log knot in angular sectors. The idea is to select an efficient segmentation method without introducing a long and complex processing step. It is important for sawmills that hope to scan logs at 2 meters per second. For biologists, the most important criterion is the precision. They want to obtain accurate measurements such as length, inclination, maximal diameter and volume of knots. The biologists and sawmills are both very interested by the exact number of knots in a log.

To fulfil this dual purpose, we have chosen to use the extraction of connected components in each angular sector based on a simple threshold. The connected components of one slice are first computed and are then merged with the ones of the previous slice.

The Fig. 7 presents several results of the connected component extraction. The main connected component of one slice is represented in pink to the left images and the corresponding 3D reconstruction is given in green on the right image. On logs without sapwood (see Fig. 7(a)), we obtain all knots with an high precision. In contrast, when logs contain sapwood (see Fig. 7(b)), the connected component extraction produces a component where the knot is connected to sapwood and growth rings. This happens when the density of sapwood and growth rings is close to the knot. Thresholding before extraction does not allow to remove them whithout distort the knot. 


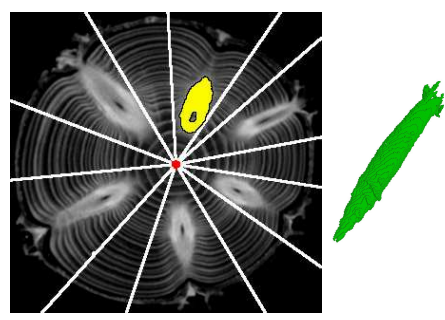

(a) Without sapwood

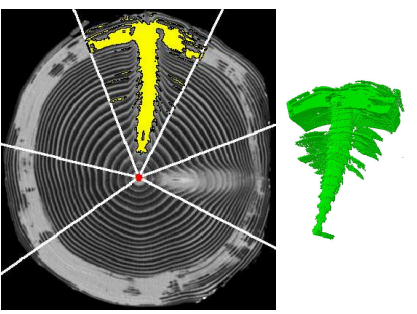

(b) With sapwood

Fig. 7. Detected angular sectors and connex components with 3D visualization.

The first parameter that our algorithm allow to compute, even with sapwood, is the number of knots. It is the number of detected angular sectors. We can also determine the inclination of all knots by extracting angle corresponding to the maximum of histogram for each angular sector (see Fig. 6). The length is determined in logs without sapwood. When there are sapwood and when a knot is connected to it, experts estimate that node leaves the stem and length can be estimate by the radius of log. The diameter can be exactly computed at any position in the $3 \mathrm{D}$ reconstruction of knots without sapwood. In the others cases, we can compute the maximum diameter because it is computed from the middle of knot and sapwood is not present at this place. Without sapwood, the volume can also be determined from the $3 \mathrm{D}$ reconstruction of knots. The sapwood induce an overestimation due to the set of parts connected to the knot.

To compare our method with other usual approaches of deformable model, we experiment one of them able to deal with automated topology changes [7]. For this purpose, the initial model was created by using a cylindrical mesh defined from the outer shape contour (see Fig. 8(a)). Note that we use the euclidean metric model implementation. The model is efficient to extract knots in logs without sapwood (See Fig. 8(a-c)). However it is not able to isolate the knot parts from sapwood areas (See Fig. 8(d)).

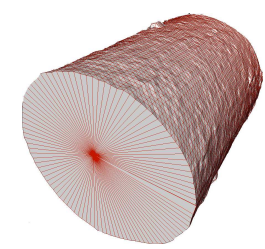

(a) Initialisation 6700 vertex

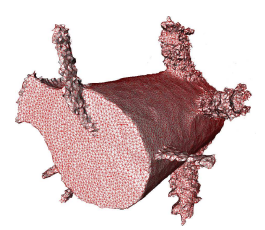

(b) 100 itera

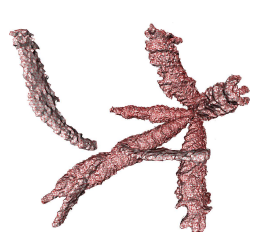

(c) 300 iterations 16206 vertex

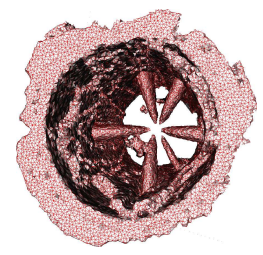

(d) 200 iterations

(with sapwood) 171934 vertex

Fig. 8. Experiments of deformable models [7] on wood with and without sapwood.

If the segmentation results are comparable for the logs without sapwood, the real advantage of our method is the computation time. We use histogram computation and extraction of connected components. The knot detection time is about the second with our approach whereas deformable model takes really 
more time, more than one hour. The proposed method is also able to detect and analyze the knots for the case of sapwood while the deformable fails to isolate such a part in this case. The source code of the proposed method is available online [8].

\section{Conclusion}

The method proposed in this paper automatically detects and counts all the knots of a given piece of wood based on the analysis of X-Ray CT images. It allows to easily deduce several measures such as inclination, length, maximal diameter and volume of knots. All its steps are based on elementary operations such as histogram computation and therefore the method is very fast. In future works, we will improve our method with objective to free ourselves of sapwood.

\section{Acknowledgements}

The authors would like to thank Jacques-Olivier Lachaud and Benjamin Taton for kindly providing the source code of the deformable model [7].

\section{References}

1. Mcinerney, T., Terzopoulos, D.: Deformable models in medical image analysis: A survey. Medical Image Analysis 1 (1996) 91-108

2. Schad, K.C., Schmoldt, D.L., Ross, R.J.: Nondestructive methods for detecting defects in softwood logs. USDA Forest Service Research paper FPL-RP-546 (1996)

3. Longuetaud, F., Mothe, F., Kerautret, B., Krähenbühl, A., Hory, L., Leban, J., Debled-Rennesson, I.: Automatic knot detection and measurements from x-ray ct images of wood: A review and validation of an improved algorithm on softwood samples. Computers and Electronics in Agriculture 85 (2012) 77-89 (to appears).

4. Longuetaud, F., Leban, J.M., Mothe, F., Kerrien, E., Berger, M.O.: Automatic detection of pith on ct images of spruce logs. Computers and Electronics in Agriculture 44 (2004) 107-119

5. Boukadida, H., Longuetaud, F., Colin, F., Freyburger, C., Constant, T., Leban, J.M., Mothe, F.: Pithextract: a robust algorithm for pith detection in computer tomography images of wood - application to $125 \operatorname{logs}$ from 17 tree species. Computers and Electronics in Agriculture 85 (2012) 90-98

6. Duda, R.O., Hart, P.E.: Use of the hough transformation to detect lines and curves in pictures. Commun. ACM 15 (1972) 11-15

7. Lachaud, J.O., Taton, B.: Deformable model with adaptive mesh and automated topology changes. In Rioux, M., Boulanger, P., Godin, G., eds.: Proc. 4th int. Conf. 3-D Digital Imaging and Modeling (3DIM'2003), Banff, Alberta, Canada, IEEE Computer Society Press (2003)

8. Krähenbühl, A.: Tkdetection : https://github.com/adrien057/TKDetection (2012) 